\title{
Infection of pancreatic pseudocyst in Candida albicans associated with Acinetobacter baumanii
}

\section{Proceeding}

Infections of acute pancreatitis are still frequent and serious complications can be bacterial or fungal origin. We report a case of infection of a pancreatic pseudocyst Candida albicans associated with Acinetobacter baumanii. 65 year old patient, admitted to the service of medical intensive care unit for management of acute pancreatitis. The examinations were performed and confirmed the presence of a gastric retro collection. Puncture pseudocyst was made for bacteriological examination which allowed the identification of Acinetobacter baumanii associated with Candida albicans. Infection of pancreatic pseudocyst by Candida albicans associated with Acinetobacter baumanii remains very rare, remains a sign of poor prognosis and should be considered in the therapeutic treatment.

\section{Introduction}

Acute pancreatitis infections are frequent complications (5 to $10 \%$ of acute pancreatitis ${ }^{1}$ and 40 to $70 \%$ of acute necrotizing pancreatitis ${ }^{2}$ and still serious ( $48 \%$ of deaths). ${ }^{3}$ These are mainly bacterial or mycotic infections with digestive origin. The association remains very rare. We report the case of infection of a Candida albicans pancreatic pseudocyst associated with a non-fermenting Gram-negative bacillus Acinetobacter baumanii.

\section{Observation}

Patient aged 65 years, chronic hemodialysis since 2012 having undergone aortic valve replacement in 2006 who has complicated arrhythmia, admitted to the service of medical resuscitation for the management of acute pancreatitis necrotico-haemorrhagic (stage E) The clinical examination found the patient in an altered general condition with diffuse abdominal tenderness. Abdominal ultrasonography showed at the level of the pancreatic box the presence of a liquid formation with heterogeneous contour with liquid-liquid level. The computed tomography (CT) performed confirmed the presence of a retro-gastric collection of $14 \times 7 \mathrm{~cm}$, hence the indication of a cystogastrostomy under echo-endoscopy. A pseudocyst puncture was made, purulent in appearance, direct examination after Gram staining showed Gram-negative bacilli and an abundance of budding yeasts. The culture and the biochemical identification made it possible to highlight Acinetobacter baumanii associated with Candida albicans. The patient was put on antifungal (Fluconazole) and antibiotic-based (Colistine) with a good improvement.
Volume 2 Issue 2 - 2018

\author{
Hicham Chemsi,' Chadli M,, Sekhsokh Y' \\ 'Equipe de Recherche Epidémiologie et Résistance bactérienne, \\ Faculté de Médecine et de Pharmacie de Rabat, université \\ Mohammed V-Souissi, Morocco \\ ${ }^{2}$ Service de bactériologie HMIMV, Faculté de Médecine et de \\ Pharmacie de Rabat, université Mohammed V-Souissi, Morocco
}

Correspondence: Hicham Chemsi, Equipe de Recherche Epidémiologie et Résistance bactérienne, Faculté de Médecine et de Pharmacie de Rabat, université MohammedV-Souissi, Maroc,Email hich100@gmail.com

Received: March 07, 2018 | Published: March 28, 2018

\section{Conclusion}

The infection of the pancreatic pseudocyst by Candida albicans associated with Acinetobacter baumanii remains very rare, and is a sign of a poor prognosis that should be taken into consideration during therapeutic treatment.

\section{Acknowledgement}

None.

\section{Conflict of interest}

The author's dealer there is no any conflict of interest.

\section{References}

1. Fedorak IJ, Ko TC, Djuricin G, et al. Secondary pancreatic infections: are they distinct clinical entities? Surgery. 1992;112:824-831.

2. Farkas G, Marton J, Mandi Y, et al. Surgical strategy and management of infected pancreatic necrosis. Br J Surg. 1996;83:930-933.

3. Aloia T, Solomkin J, Fink AS, et al. Candida in pancreatic infection: a clinical experience. Am Surg. 1994;60(10):793-796. 